\title{
PLASMA PROCESSES IN THE OUTER COMA
}

\author{
A. A. GALEEV \\ Space Research Institute of the USSR Academy of Sciences \\ 117810, GSP-7, Moscow, USSR
}

\begin{abstract}
Spacecraft encounters with comets Giacobini-Zinner and Halley revealed a great variety of collective plasma phenomena accompanying the interaction of the solar wind with comets. In this review, we discuss the theory and in situ measurements of the Alfven wave turbulence and the solar wind loading by cometary ions, and the structure of the cometary bow shock.
\end{abstract}

\section{Introduction}

Bierman et al. (1967) introduced the concept of the solar wind loading by photoionized atoms from the cometary atmosphere. This concept has changed completely all the previous theoretical models of the solar wind interaction with comets, which have been based on the assumed analogy to the solar wind interaction with planets.

Bierman et al. (1967) have shown that, in contrast to the solar wind interaction with planets, the interaction with freely expanding cometary atmospheres has already started at distances of millions of kilometers from the tiny cometary nucleus. At these distances, the cometary atmospheres are ionized by solar light or through the charge exchange with solar wind ions. This interaction results in the formation of a cometary bow shock with a standoff distance to the cometary nucleus on the order of several hundreds of thousands of kilometers (see Figure 1). Long before the encounters of space probes with comets in 1985-1986, numerous instabilities were discovered accompanying the mass loading of the solar wind by newly created ions (Hartle and Wu (1973), Wu and Davidson (1972), Wu and Hartle (1974)). However, nobody expected that a relatively small number of newly created ions would generated such strong solar wind magnetohydrodynamic (MHD) turbulence in a huge area about the comet as was discovered first near the Giacobini-Zinner comet (Smith et al. (1986), Bame et al. (1986), Scarf et al. (1986)). After this discovery, Sagdeev et al. (1986) immediately developed the quasi-linear theory of Alfvén wave generation by cometary ions that is adequate upstream, but not far from the cometary bow shock $\left(\leq 2.6 \times 10^{6} \mathrm{~km}\right.$, Coates et al. $\left.(1989 \mathrm{~b})\right)$, where the energy of excited Alfven waves is still lower than the densities of magnetic field energy and thermal plasma energy. Encounters of the VEGA and Giotto spacecraft with comet Halley provided considerable new data on MHD turbulence near comets. In particular, it was confirmed that, at large distances from the comet, Alfvén waves dominate the spectrum of MHD turbulence (Johnstone et al. (1987)), and the spectra of Alfvén wave turbulence (Glassmeier et al. (1989)) are in a good agreement with the predictions of the quasi-linear theory (Sagdeev et al. (1986), Galeev et al. (1987b) and happen to be different from the Kolmogorov spectra of MHD turbulence near comet Giacobini-Zinner (Tsurutani and Smith (1986a)). We describe the quasi-linear theory and in situ measurements of MHD turbulence in Section 2 of our review.

In Section 3, to describe the flow of solar wind loaded by cometary ions, we use the semikinetic 


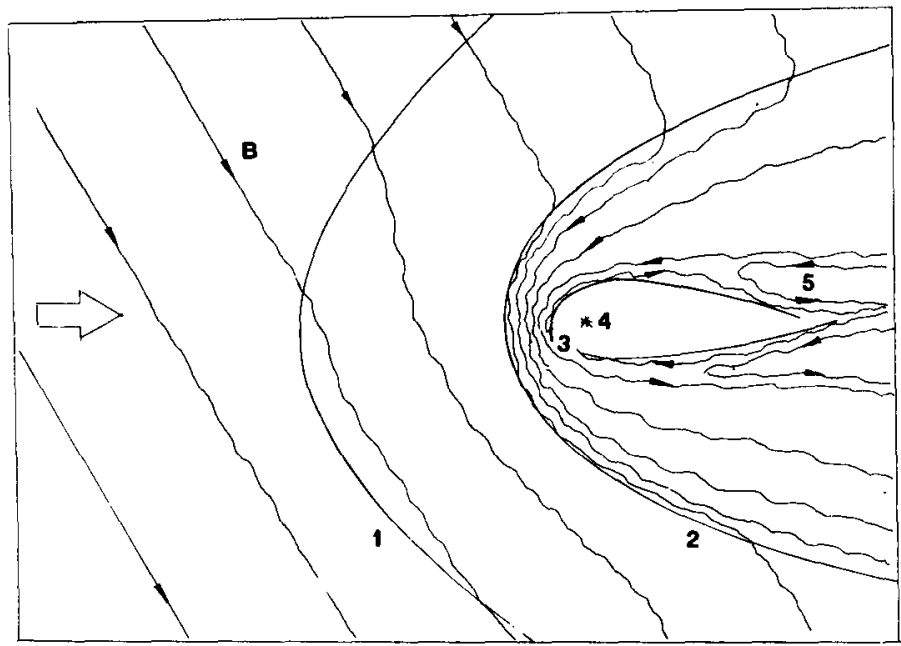

Figure 1. Schematic picture of the solar wind-comet interaction and characteristic plasma boundaries: the bow shock (1), the cometopause (2), and the contact surface (3).

approach with a kinetic equation for cometary ions and the hydrodynamic equation for the solar wind (Wallis and Ong (1975), Galeev et al. (1985a)). This approach permits us to calculate the stochastic acceleration of cometary ions by MHD waves (Ip and Axford (1986), (1987), Gribov et al. (1986), Isenberg (1987), Gombosi (1988), Barbosa (1989)).

Spacecraft measurements in the cometary bow shocks have provided detailed data on the bow shocks' structure. These measurements not only have resolved the long-standing controversy about the existence of the bow shock (Bierman et al. (1967), Wallis (1971)), but have also stimulated a new round of computer simulations (Omidi and Winske $(1987,1988)$, Lipatov et al. (1989)) to study the effect of MHD turbulence and the interplanetary magnetic field on the existence of the subshock predicted by earlier simulations (Galeev et al. (1985b)) for the perpendicular cometary shock. Here we present the measurements and discuss the structure of cometary bow shocks in terms of the isothermal jump in the shock front (Landau and Lifshitz (1988)).

\section{Alfvén Wave Turbulence Near Comets}

On the basis of earlier analyses of plasma instabilities due to the ionization of neutral atoms in the solar wind flow (Hartle and Wu (1973), Wu and Davidson (1972), Wu and Hartle (1974), Winske et al. (1985), Gary et al. (1984)), it was already clear before the spacecraft encounters with comets that both MHD and lower-hybrid waves should be generated in the vicinity of comets by locally born cometary ions (Ip and Axford (1982)). But only after the encounter of the International Cometary Explorer (ICE) spacecraft with comet Giacobini-Zinner could we concentrate our efforts on the theory of Alfvén wave generation and their back reaction on the velocity distribution of cometary ions, since the MHD waves were proven to be the most intense and the Alfvén waves are excited more easily than the magnetosonic waves that were damping heavily in the finite $\beta$ solar wind plasma. As a result, the quasi-linear theory of Alfvén wave generation and solar wind loading by cometary 
ions was developed by Sagdeev et al. (1986) and later elaborated in greater detail (Galeev et al. (1987b), Galeev and Sagdeev (1988)). Unfortunately, there is no comprehensive nonlinear theory of MHD wave generation that would adequately describe the nonlinear wave forms discovered by the ICE spacecraft near comet Giacobini-Zinner (Tsurutani and Smith (1986b), Tsurutani et al. 1987)). Therefore, here we limit ourselves to the quasi-linear theory of Alfvén wave generation and its comparison with the observations near comet Halley.

\subsection{DIFFUSION SHELLS APPROACH TO THE COMETARY ION VELOCITY DISTRIBU- TION AND WAVE GENERATION}

As is known, the reaction of excited waves on the velocity distribution of the plasma particles can be reduced to a diffusion equation for the resonant particles in velocity space (Sagdeev and Galeev (1969)). But before going into the derivation and analysis of this equation for the cometary ion velocity distribution, we show in this subsection that many results of the quasi-linear theory could be easily obtained without solving this equation in the particular case when all excited Alfven waves propagate along the magnetic field lines (Galeev and Sagdeev, 1988)). The latter can be justified by noting that these waves have a larger growth rate than the obliquely propagating waves (Winske et al. (1985)) and therefore, far from the comet, where the growth rate is small, only these waves can grow.

Simplification comes from the fact that, in the system of coordinates moving with the Alfven wave, the wave electric field is zero and, as a result, the energy of particles interacting with this wave is conserved. Therefore, if the particles in the process of diffusion in velocity space interact only with waves propagating in the same direction, and therefore having the same phase velocity, their energy is conserved in the system of coordinates moving with the phase velocity of Alfvén waves, i.e., particles diffuse along the spherical shell in velocity space centered on the phase velocity of Alfvén waves.

Using this property of the Alfven wave-particle interaction, we show graphically in Figure 2 how the velocity of locally born cometary ions changes under the back reaction of Alfvén waves generated by these ions. In the system of coordinates moving with the plasma that is used to draw this figure, the locally born cometary ions initially form in velocity space a ring with radius $u \sin \alpha$ in the plane perpendicular to the $v_{\|}$axis and shifted along this axis by the value $v_{\|}=-u \cos \alpha$ relative to the origin of coordinate system. Here we assume that the solar wind flows with velocity $u(x)$ in a cometary system of coordinates along the $\mathrm{x}$-axis directed towards the nucleus of a comet, and the vector $\mathbf{B}$ of the interplanetary magnetic field forms the angle $\alpha$ with the direction of plasma flow. Locally ionized cometary atoms in the cometary system of coordinates have the initial velocity $V_{g} \approx 1 \mathrm{~km} / \mathrm{s}$ of the cometary gas evaporated from the cometary nucleus by the solar radiation that will be neglected here in comparison with the solar wind velocity. As a result, in the system of coordinate moving with plasma, the ionized cometary atoms start to rotate along the cyclotron orbit with the velocity $u \sin \alpha$ equal to the component of its total velocity $-\mathbf{u}$ perpendicular to the magnetic field, and the ionized cometary atoms continue to move along the magnetic field with the gas velocity $-u \cos \alpha$ in this system. In Figure 2, which represents the cut of the velocity space by the plane containing the longitudinal velocity axis, the ring of cometary ions is shown by two dots with the coordinates $(-u \cos \alpha, \pm u \sin \alpha)$ in the $\left(v_{\|}, v_{\perp}\right)$ plane. According to the aforementioned properties of the Alfven wave-cometary ion interaction, the ions can diffuse from the initial ring along the surfaces of two spheres with the centers on the $v_{\|}$axis at the points $v_{\|}=+V_{\mathrm{A}}$ or $v_{\|}=-V_{\mathrm{A}}\left(V_{\mathrm{A}}\right.$ is the Alfven velocity), depending on the direction of the propagation of waves interacting with ions (in the direction of the vector $\mathbf{B}$ or in the opposite direction, respectively). If we draw in Figure 2 the sphere of constant ion energy $v_{\|}^{2}+v_{\perp}^{2}=u^{2}$ through the initial ring of cometary ions, then we see that in order to excite Alfvén waves, the cometary ions have to diffuse towards lower energies, i.e., in the velocity regions $v_{\|}<-u \cos \alpha$ and $v_{\|}>-u \cos \alpha$ they diffuse along the sphere centered at $v_{\|}=V_{\mathrm{A}}$ and $v_{\|}=-V_{\mathrm{A}}$, respectively (see Figure 2). The value of the wave vector $\mathbf{k}$ and the sign 
of the polarization of waves generated by the cometary ions with the velocity $v_{\|}$are found from the condition of cyclotron resonance between waves and particles

$$
\omega_{k}-k v_{\|} \pm \omega_{c i}=0
$$

where $\omega_{c i}=e_{i} B / m_{i c}$ is the cyclotron frequency of cometary ions with charge $e_{i}$ and mass $m_{i}$, and the wave frequency $\omega_{k}$ is considered to be positive. The signs "+" and " - " refer to the right- and left-hand polarization of the wave, respectively (the magnetic field vectors $\mathbf{B}_{k}^{(+)}$and $\mathbf{B}_{k}^{(-)}$rotate in the directions of cyclotron motion of electrons and ions, respectively). The polarization of excited waves and the direction of propagation are also indicated in Figure 2, assuming that the angle $\alpha<90^{\circ}$ and thus the positive (negative) wave vector $\mathrm{k}$ correspond to the antisunward (sunward) wave propagation.

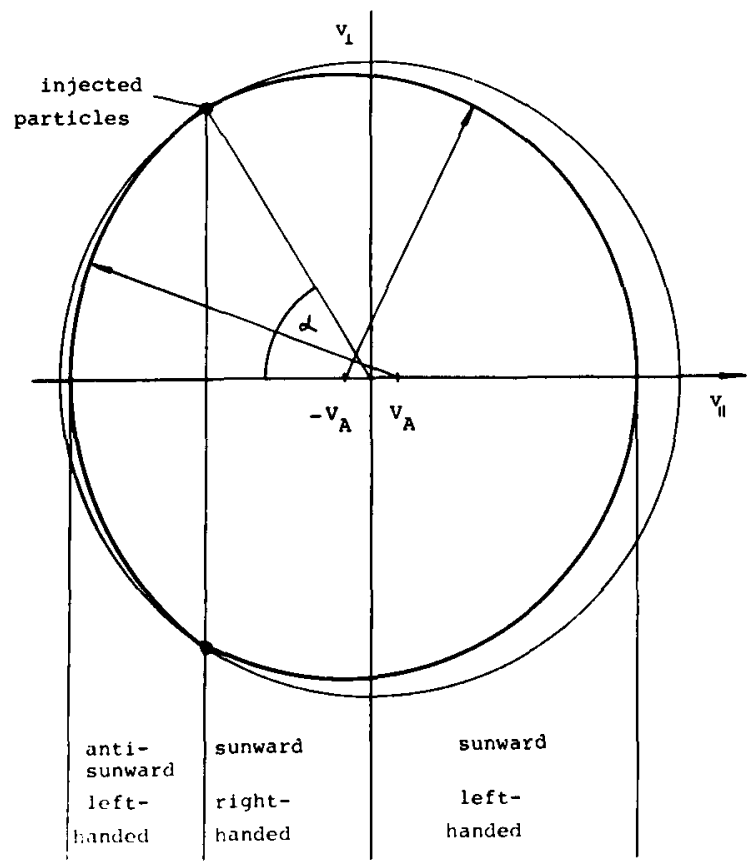

Figure 2. The cross section of diffusion surfaces (thick line) for the diffusion of cometary ions in velocity space under the action of Alfvén waves excited by the cometary ions born in a solar wind flow (injected particles). The direction of propagation and the polarization of resonant Alfvén waves are specified for the different velocity space regions (from Galeev and Sagdeev (1988)).

The fraction of the energy of cometary ions transferred to excited Alfvén waves can be easily found assuming that, as a result of scattering by waves, the cometary ions injected on the ring in the velocity space are spread uniformly over the two hemispherical surfaces intersecting each other at this ring. The relative fraction of energy transferred to waves is given by the expression

$$
\varepsilon(\alpha)=1-\frac{\left(\int v^{2} d S_{-}+\int v^{2} d S_{+}\right)}{u^{2}\left(\int d S_{-}+\int d S_{+}\right)} \approx\left(V_{\mathrm{A}} / u\right)\left(1+\cos ^{2} \alpha\right)
$$


where the integration over the hemispheres $S_{+}$and $S_{-}$can be reduced to the integration over the respective polar angle $\mu=\cos \theta$,

$$
\begin{aligned}
d S_{ \pm} & =2 \pi v_{ \pm}^{2} d \mu_{ \pm}, \quad v_{ \pm}^{2}=u^{2} \sin ^{2} \alpha+\left(u \cos \alpha \pm V_{\mathrm{A}}\right)^{2} \\
-1 & \leq \mu_{+} \leq-\left(u \cos \alpha+V_{\mathrm{A}}\right) / v_{+}, \quad-\left(u \cos \alpha-V_{\mathrm{A}}\right) / v_{-}<\mu_{-}<1
\end{aligned}
$$

Here we have assumed that cometary ions are locally born and thus their injection velocity is equal to the local solar wind velocity $u(x)$, which does not change significantly at far distances from the comet. To calculate the value of $\varepsilon(\alpha)$, we neglected higher-order terms in the small parameter $V_{\mathrm{A}} \ll 1$, which is also done to solve the quasi-linear equations in the next subsection. There we obtain the more general result taking into account the injection of cometary ions into the solar wind not only due to the local ionization of cometary gas, but also due to their diffusion along magnetic field lines from the downstream regions. However, this additional injection source is numerically so small that it only slightly corrects the numerical value of $\varepsilon(\alpha)$ given by Equation (2) and does not change the diffusion pattern shown in Figure 2.

Another interesting property of the uniform distribution of cometary ions over the two hemispheres in Figure 2 resulting from the strong pitch angle scattering of cometary ions by Alfvén waves is the residual flow of cometary ions relative to the solar wind along the magnetic field lines. The velocity of this flow can be expressed through the integrals over these hemispheres:

$$
\delta u_{\|}=\frac{\left(\int v_{\|} d S_{+}+\int v_{\|} d S_{-}\right)}{\left(\int d S_{+}+\int d S_{-}\right)} \approx-\frac{3}{2} V_{\mathrm{A}} \cos \alpha\left(1-\frac{1}{3} \cos ^{2} \alpha\right),
$$

where we again neglected the higher-order terms in the small parameter $V_{\mathrm{A}} / u \ll 1$. Let us note that this expression for the bulk velocity of cometary ions is more general than that given by the quasi-linear theory, since it could be used for the arbitrary ratio of $V_{\mathrm{A}} / U<1$. However, in the limit $V_{\mathrm{A}} / U \ll 1$ both expressions give the same value of $\delta u_{\|}$.

\subsection{THE SOLUTION OF THE QUASI-LINEAR EQUATION}

The quasi-linear equation for the velocity distribution of cometary ions we write here combines the general kinetic equation in the drift approximation (Kulsrud (1983)), the well-known expression for the quasi-linear diffusion of ions (Sagdeev and Galeev (1969)), and the source term describing the local ionization of cometary atoms (Sagdeev et al. (1986)):

$$
\begin{aligned}
& \left(u+v_{\|} \cos \alpha\right) \frac{\partial f}{\partial x}+\frac{v_{\perp}^{2}}{2 B}\left(u+v_{\|} \cos \alpha\right) \frac{d B}{d x} \frac{\partial f}{v_{\perp} \partial v_{\perp}}- \\
& \quad-\left[\frac{v_{\perp}^{2}}{2 B} \frac{d B}{d x}-\left(u+v_{\|} \cos \alpha\right) \frac{d u}{d x}\right] \cos \alpha \frac{\partial f}{\partial v_{\|}}= \\
& \quad=\frac{\pi e_{i}^{2}}{m_{i}^{2}} \int \frac{d k}{2 \pi} \sum_{ \pm}\left[\left(1-\frac{k v_{\|}}{\omega_{k}}\right) \frac{1}{v_{\perp}} \frac{\partial}{\partial v_{\perp}} v_{\perp}+\frac{k v_{\perp}}{\omega_{k}} \frac{\partial}{\partial v_{\|}}\right]\left|B_{k}^{ \pm}\right|^{2} \times \\
& \quad \times\left(\frac{\omega_{k}}{k c}\right)^{2} \delta\left(\omega_{k}-k v_{\|} \pm \omega_{c i}\right)\left[\left(1-\frac{k v_{\|}}{\omega_{k}}\right) \frac{\partial}{\partial v_{\perp}}+\frac{k v_{\perp}}{\omega_{k}} \frac{\partial}{\partial v_{\|}}\right] f+ \\
& \quad+(\dot{N} / \pi) \delta\left(v_{\perp}^{2}-u^{2} \sin ^{2} \alpha\right) \delta\left(v_{\|}+u \cos \alpha\right) .
\end{aligned}
$$

The first term on the left-hand side describes the hydrodynamic convection of cometary ions by plasma and their free motion along magnetic field lines. The second accounts for the adiabatic heating of cometary ions. In the third term, we included, along with the magnetic mirror force, the inertia force (the second term in the square brackets) that was erroneously omitted in the previous calculations (Galeev and Sagdeev (1988)). The last term in Equation (5) describes the injection of 
cometary ions into the solar wind with the rate $\dot{N}$ defined by the gas production by a comet $Q$, the velocity $V_{g}$ of spherical expansion of a gas, and the time $\tau$ for gas ionization

$$
\dot{N}=\left(Q / 4 \pi V_{g} \tau r^{2}\right) \exp \left(-r / V_{g} \tau\right)
$$

where $r$ is the distance to the nucleus of a comet ( $r=-x$ along the flow line through the nucleus). The exponential factor here could be neglected only if the bow shock distance to the comet nucleus is smaller than the ionization length $V_{g} \tau$.

For the sake of convenience in further calculations, we rewrite Equation (5) in a spherical system of coordinates in velocity space with the polar axis along the magnetic field

$$
\begin{aligned}
\frac{\partial}{\partial x}(u & +\mu v \cos \alpha) f-\frac{1}{v^{2}} \frac{\partial}{\partial v} v^{2}\left[\frac{1}{3} \frac{d u}{d x} v f+A \frac{\partial f}{\partial \mu}\right]+ \\
& +\frac{\partial}{\partial \mu}\left[\frac{1}{2}\left(1-\mu^{2}\right) \frac{d \cos \alpha}{d x} v f+A \frac{\partial f}{\partial v}\right]=\operatorname{St}_{\mathrm{QL}}(f)+ \\
& +\left(\dot{N} / 2 \pi v^{2}\right) \delta(v-u) \delta(\mu+\cos \alpha)
\end{aligned}
$$

where

$$
\begin{gathered}
A=\frac{1}{2}\left[u \cos \alpha-\mu v\left(\sin ^{2} \alpha-\frac{2}{3}\right)\right]\left(1-\mu^{2}\right) \frac{d u}{d x} \\
\operatorname{St}_{\mathrm{QL}}(f)=\frac{\partial}{\partial \mu}\left(1-\mu^{2}\right)\left[\nu_{\mu \mu} \frac{\partial f}{\partial \mu}+\nu_{\mu v} v \frac{\partial f}{\partial v}\right]+\frac{1}{v^{2}} \frac{\partial}{\partial v} v^{3}\left(1-\mu^{2}\right)\left[\nu_{v \mu} \frac{\partial f}{\partial \mu}+\nu_{v v} v \frac{\partial f}{\partial v}\right] \\
\nu_{\alpha \beta}=\frac{\pi e_{i}^{2}}{m_{i}^{2} c^{2}} \int \frac{d k}{2 \pi} \sum_{ \pm}\left|B_{k}^{ \pm}\right|^{2} \delta\left(\omega_{k}-k v \mu \pm \omega_{c i}\right) A_{\alpha \beta}=\sum_{ \pm} \nu_{ \pm} A_{\alpha \beta} \\
A_{\mu \mu}=\left(1-\frac{\omega_{k} \mu}{k v}\right)^{2} \quad A_{\mu v}=A_{v \mu}=\frac{\omega_{k}}{k v}\left(1-\frac{\omega_{k} \mu}{k v}\right) \quad A_{v v}=\left(\frac{\omega_{k}}{k v}\right)^{2} .
\end{gathered}
$$

In order to express the magnetic field gradient through the plasma flow velocity gradient, we have used here the equations of the ideal MHD flow

$$
\operatorname{div} \mathbf{B}=0 \text { and } \operatorname{rot}[\mathbf{u} \times \mathbf{B}]=0 \text { eqno(11) }
$$

Neglecting small $y, z$ components of plasma flow velocity (Baranov et al. (1986)) we have obtained

$$
(d / d x) \ln B=-\sin ^{2} \alpha(d / d x) \ln u=-(d / d x) \ln \cos \alpha
$$

Let us note that in the superalfvenic solar wind flow, the pitch-angle scattering of cometary ions by Alfvén waves is much faster than their diffusion in energy. This statement is easily proved by the ordering of the rates (Equation (10)) of corresponding processes described by the quasi-linear collision term (Equation (9))

$$
\nu_{\mu \mu}: \nu_{\mu v}: \nu_{v \mu}: \nu_{v v}=1:\left(V_{\mathrm{A}} / v\right):\left(V_{\mathrm{A}} / v\right):\left(V_{\mathrm{A}} / v\right)^{2}
$$

Therefore, in the first approximation, the velocity distribution of cometary ions can be considered isotropic in velocity space and written in the form

$$
f(x, v, \mu)=f_{0}(x, v)+f_{1}(x, v, \mu),
$$

where $f_{1}$ describes the small anisotropy on the order of $\left(V_{\mathrm{A}} / v\right)$. Upstream of the cometary bow shock, the amplitude of magnetic field oscillations in Alfven waves is only a few times lower than the ambient magnetic field strength (Smith et al. (1986), Tsurutani and Smith (1986a) (1986b), 
Johnstone et al. (1987), Glassmier et al. (1989)). Therefore, the frequency $\nu_{\mu \mu}$ of the pitch angle scattering of cometary ions is on the order of the gyrofrequency, and the quasi-linear collisional term (Equation (9)) dominates in Equation (7). Retaining in the latter only the lower-order terms in the $\left(V_{\mathrm{A}} / v\right)$ ratio, we obtain the small anisotropic part of the ion velocity distribution

$$
\partial f_{1} / \partial \mu=-\left(v \nu_{\mu v} / \nu_{\mu \mu}\right) \partial f_{0} / \partial v .
$$

This equation, in fact, describes the cometary ion velocity shells that are displaced along the magnetic field lines by the Alfven wave phase velocity (see Figure 2). With this result, we can now obtain the kinetic equation for the isotropic part of the ion velocity distribution function by the averaging of Equation (7) over the pitch-angles

$$
\begin{aligned}
\frac{\partial}{\partial x}\left[u f_{0}\right. & \left.-\frac{1}{2} \cos \alpha\left\langle\left(1-\mu^{2}\right) \frac{\nu_{\mu v}}{\nu_{\mu \mu}}\right\rangle v^{2} \frac{\partial f_{0}}{\partial v}\right]-\frac{1}{v^{2}} \frac{\partial}{\partial v} v^{2}\left(\frac { 1 } { 3 } \frac { d u } { d x } \left\{v f_{0}\right.\right. \\
& \left.\left.-\frac{3}{2}\left\langle\left[u \cos \alpha-\mu v\left(\sin ^{2} \alpha-\frac{2}{3}\right)\right]\left(1-\mu^{2}\right) \frac{\nu_{\mu v}}{\nu_{\mu \mu}}\right\rangle v \frac{\partial f_{0}}{\partial v}\right\}\right) \\
& =\left(\dot{N} / 4 \pi v^{2}\right) \delta(v-u),
\end{aligned}
$$

where the angular brackets mean the averaging over the pitch angles

$$
\left\langle\psi(\mu) \frac{\nu_{\mu v}}{\nu_{\mu \mu}}\right\rangle=\frac{1}{2} \int_{-1}^{+1} \psi(\mu) \frac{\nu_{\mu v}}{\nu \mu \mu} d \mu
$$

Let us note that Equation (16) is very similar to the kinetic equation for the cosmic rays interacting with Alfvén waves (see the review by Forman and Webb (1985)). If we assume that the locally excited Alfvén waves dominate the Alfvén wave turbulence, then we can further specify the averaging procedure

$$
\left\langle\psi(\mu) \frac{\nu_{\mu v}}{\nu_{\mu \mu}}\right\rangle=\frac{V_{\mathrm{A}}}{2 v}\left[\int_{-u / v}^{-u \cos \alpha / v} \psi(\mu) d \mu-\int_{-u \cos \alpha / v}^{u / v} \psi(\mu) d \mu\right]
$$

Here we have taken into account that, according to our discussion in the previous subsection, the cometary ions with the given parallel velocity $\mu v$ interact with the Alfven wave that has the specific polarization and direction of propagation (see Figure 2). Therefore, the ratio of the given above "collision" frequencies $\nu_{\mu \nu} / \nu_{\mu \mu}$ is equal to the ratio of the parallel phase velocity of resonant waves and the particle absolute velocity. With this averaging procedure, we can verify that, in the velocity shell approximation, the ions are moving through the solar wind along the magnetic field lines with the bulk velocity

$$
\delta u_{\|}=\frac{3}{2} v\left\langle\left(1-\mu^{2}\right) \frac{\nu_{\mu \nu}}{\nu_{\mu \mu}}\right\rangle=-\frac{3}{2} V_{\mathrm{A}} \cos \alpha\left(1-\frac{1}{3} \cos ^{2} \alpha\right)
$$

that coincides with our earlier calculations (Equation (4)).

The residual anisotropy of the cometary ion velocity distribution caused by the nonuniform plasma convection, adiabatic heating and ionization of cometary atoms far from the comet is very small. Nevertheless, it is this anisotropy that results in the Alfvén wave excitation and therefore we have to retain in Equation (7) the convection, ionization, and adiabatic heating terms in spite of the fact that the resulting corrections are small (compare with Equation (15))

$$
\begin{aligned}
\nu_{\mu \mu} \frac{\partial f_{1}}{\partial \mu} & =-\nu_{\mu \nu} v \frac{\partial f_{0}}{\partial v}-\frac{\dot{N}}{2 \pi v^{2}} \frac{\delta(v-u)}{\left(1-\mu^{2}\right)}[\eta(\mu+\cos \alpha)-(1+\mu) / 2]- \\
& -(v / 2) \cos \alpha \frac{\partial f_{0}}{\partial x}-\frac{1}{2}\left[\mu v\left(\sin ^{2} \alpha-\frac{2}{3}\right)-u \cos \alpha\right] \frac{d u}{d x} \frac{\partial f_{0}}{\partial v}
\end{aligned}
$$


where $\eta(x)=1$ for $x \geq 0$ and $\eta(x)=0$ for $x<0$.

The growth rate $\gamma_{k}$ of Alfvén waves can be easily found from the energy conservation law in the system "cometary ions + Alfvén waves" in the form

$$
2 \pi \int_{0}^{\infty} v^{2} d v \int_{-1}^{+1} d \mu\left(m_{i} v^{2} / 2\right) \operatorname{St}_{\mathrm{QL}}(f)+\sum_{ \pm} \int \frac{d k}{2 \pi} 2 \gamma_{k}^{ \pm} \frac{\left|B_{k}^{ \pm}\right|^{2}}{4 \pi}=0
$$

Using Equation (9) for the quasi-linear collisional term, we obtain from Equation (21) the well-known result (Sagdeev and Shafranov (1961), Wentzel (1974), Bell (1978))

$$
\begin{aligned}
\gamma_{k}^{ \pm} & =\frac{4 \pi^{3} \omega_{k} e_{i}^{2}}{k m_{i}} \int_{0}^{\infty} v^{2} d v \int_{-1}^{+1} d \mu\left(1-\mu^{2}\right)(v / c)^{2} \delta\left(\omega_{k}-k v \mu \pm \omega_{c i}\right) \times \\
& \times\left[\left(1-\mu \frac{\omega_{k}}{k v}\right) \frac{1}{v} \frac{\partial f_{1}}{\partial \mu}+\frac{\omega_{k}}{k v} \frac{\partial f_{0}}{\partial v}\right]
\end{aligned}
$$

Combining equations (20) and (22) and using delta function approximation for the cometary ions distribution $f_{0}$ over the thin ion shell $(u \gg|r d u / d x|)$ in the velocity space, we finally obtain (Galeev et al. (1987b))

$$
\gamma_{k}^{ \pm}=\frac{2 \pi \omega_{k} \omega_{c i}}{k^{3}} \cdot \frac{\dot{N} m_{i}}{\left|B_{k}^{ \pm}\right|^{2}}\left\{\left(1 \pm \frac{\omega_{c i}}{k u_{\infty}}\right)-2 \eta\left(\cos \alpha \pm \frac{\omega_{c i}}{k u_{\infty}}\right)-\frac{1}{2} \cos \alpha\left(1-\frac{\omega_{c i}^{2}}{k^{2} u_{\infty}^{2}}\right)\right\}
$$

where $u_{\infty}$ is the solar wind flow velocity far from the comet. Here in the approximation of a thin ion shell in velocity space, the contribution of the last term in Equation (20) to the growth rate (Equation (23)) can be neglected (for details, see Galeev et al. (1987b)). With the help of Equation (23), we can now prove the main conclusion of our qualitative analysis in subsection 2.1 , that the ions with the given resonant velocity $\mu v= \pm \omega_{c i} / k$ generate only Alfvén waves with the definite direction of propagation and polarization indicated in Figure 2. This is because the main contribution to the Alfven wave generation comes from the locally born ions and the contribution of the ions coming here along the magnetic field lines is always smaller, but comparable numerically.

The spectrum of Alfvén waves is well-defined in the quasi-linear theory and could be easily calculated from the equation of wave growth in the form

$$
\left[u+\left(\omega_{k} / k\right) \cos \alpha\right] \frac{d}{d x}\left|B_{k}^{ \pm}\right|^{2}=2 \gamma_{k}^{ \pm}\left|B_{k}^{ \pm}\right|^{2}
$$

where the small term describing the spontaneous emission by pickup ions was neglected.

In order to compare the theoretical spectrum with the observations, we introduce here the spectral energy density of Alfvén waves in frequency space instead of in wave vector space

$$
\begin{aligned}
\left|B_{f}\right|^{2} & \equiv \sum_{ \pm} \sum_{k= \pm|k|}\left|B_{k}^{ \pm}\right|^{2} \frac{d|k|}{2 \pi d f}= \\
& =2 \pi \int_{-\infty}^{x} \dot{N} m_{i} u_{\infty} V_{\mathrm{A}} d x \cdot \frac{f_{c i}}{f^{2}}\left\{2\left[1-\frac{f_{c i}}{f}\right] \eta\left(\frac{f_{c i}}{|\cos \alpha|}-f\right) \eta\left(f-f_{c i}\right)+\right. \\
& \left.+\left[2+|\cos \alpha|\left(1-\frac{f_{c i}^{2}}{f^{2}}\right) \eta\left(f-\frac{f_{c i}}{|\cos \alpha|}\right)\right]\right\}
\end{aligned}
$$

where $f \simeq|k| u_{\infty}$ is the Doppler-shifted frequency of Alfvén wave. Here we have added intensities of waves with different polarizations and directions of propagation, but with the same Doppler-shifted frequency. 
The frequency integrated energy density of linearly growing Alfvén waves is proportional to the kinetic energy of injected cometary ions

$$
\frac{1}{4 \pi} \int_{f_{c i}}^{\infty}\left|B_{f}\right|^{2} d f \simeq \frac{V_{\mathrm{A}}}{u_{\infty}}\left[1+2 \cos ^{2} \alpha-\frac{1}{3} \cos ^{4} \alpha\right] \int_{-\infty}^{x} \frac{\dot{N} d x}{u_{\infty}} \cdot \frac{m_{i} u_{\infty}^{2}}{2}
$$

Comparing this equation with Equation (2), we see that the ions produced closer to the comet and then leaking along the magnetic field lines to the upstream region make the intensity of locally excited Alfvén waves higher.

Let us note finally that the approximation of strong pitch-angle scattering could fail not because the scattering frequency is low, but because there is the gap in the pitch-angle diffusion in the region of resonant velocities of thermal protons where the waves strongly damp

$$
v_{\|}<\delta v_{*}=\frac{3 m_{p}}{m_{i}} v_{T p}
$$

Here $v_{T p}$ is the thermal velocity of protons. The easiest way to go through this gap is by trapping cometary ions between the magnetic mirrors formed by the resonant Alfvén wave magnetic field $\mathbf{B}_{\mathbf{k}} \perp \mathbf{B}_{\mathbf{0}}$. The condition for this has the form

$$
\frac{m_{i} v_{\perp}^{2}}{2 B_{0}} \delta B \simeq \frac{m_{i} v_{\perp}^{2}}{2} \frac{\delta v_{*} \int B_{f}^{2} d f}{2 v B_{0}^{2}}>\frac{m_{i} \delta v_{*}^{2}}{2}
$$

where the small factor $\left(\delta v_{*} / v\right)$ on the left-hand side of the inequality accounts for the fraction of resonant waves in the total energy density of waves. Using Equation (26), we rewrite this condition as

$$
\frac{\pi m_{i} V_{\mathrm{A}} \int \dot{N} d x}{B_{0}^{2}}\left(1+2 \cos ^{2} \alpha-\frac{1}{3} \cos ^{4} \alpha\right)>\frac{3 m_{p}}{m_{i}}
$$

Computer simulations (Galeev et al. (1987a), Gary et al. (1989), Winske et al. (1985)) usually do not exhibit very isotropic ion distributions, even in this limit; that could be partially due to not exact scaling of parameters.

\subsection{OBSERVATIONS VERSUS QUASI-LINEAR THEORY}

We have used two approximations that greatly simplified solution of the quasi-linear equations: the approximation of a thin cometary ion shell in velocity space and the approximation of strong pitch angle scattering that maintains isotropy of ion distribution within this shell. Measurements aboard the Suisei spacecraft (Mukai et al. (1986)) have clearly shown that the cometary ion distribution is not uniform within its shell. Later analysis of the measurements of the three-dimensional velocity distribution of cometary protons (Neugebauer et al. (1987), (1989a), (1989b)) and heavy ions (Coates et al. (1989a), (1989b)) provided a detailed description of the ion shell structure. From the cuts of the cometary proton shell shown in Figure 3, we see that most of the protons retain their initial velocity without much pitch angle scattering. Coates et al. (1989a) found that the velocity of cometary ions along the magnetic field lines far from the shock strongly correlates with the injection velocity

$$
u_{\|}=K u \cos \alpha
$$

where the correlation factor $K$ is typically between 0.5 and 1.5 . Only close to the cometary bow shock $\left(<2.6 \times 10^{6} \mathrm{~km}\right.$ according to Coates et al. (1989b)), where the condition (Equation (29)) for strong pitch-angle scattering and effective mirroring is satisfied, does the correlation factor drop to 


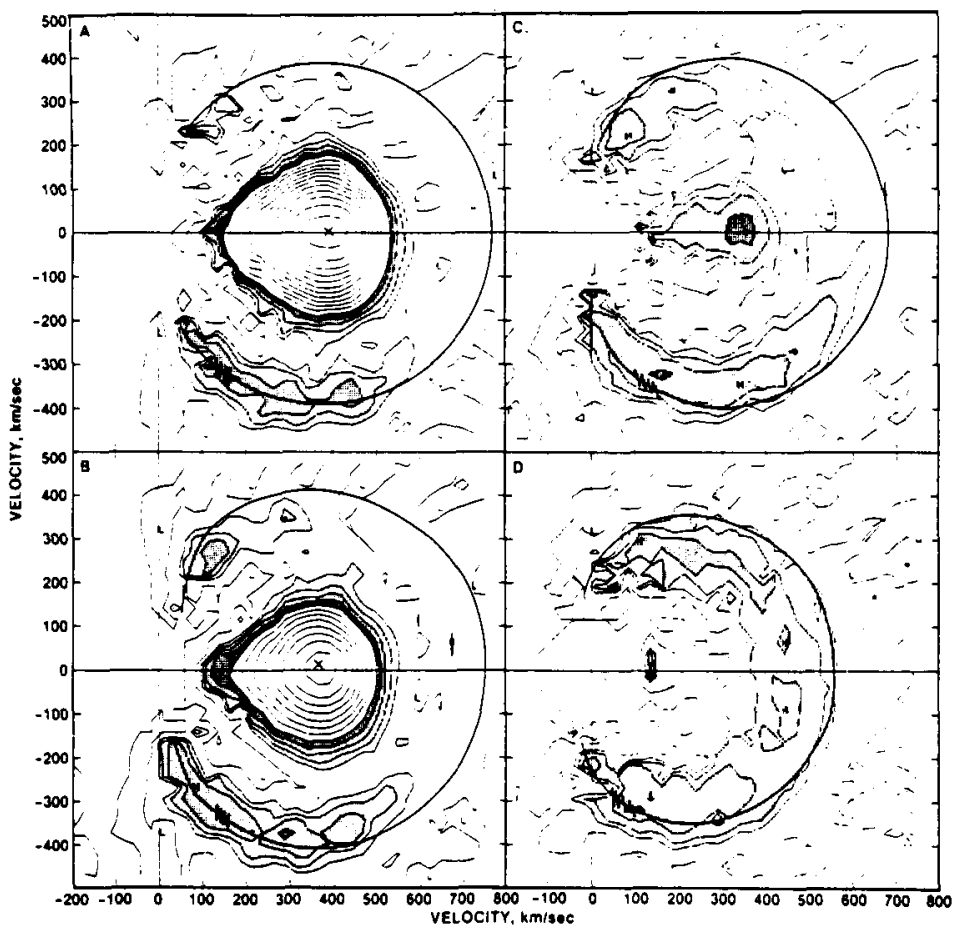

Figure 3. Polar plots of contours of the proton phase-space density $f(v)$ on a conical surface in velocity space viewed by the four channel electron multiplier detectors A, B, C and D on the spinning Giotto spacecraft (from Neugebauer et al. (1987)).

about $K \sim v_{A} / u$. This is in qualitative agreement with our estimate (Equation (4)) in the limit of strong pitch-angle scattering.

From these observations we can conclude that the approximation of the thin ion shell is reasonable, but the anisotropy of the ion distribution over this shell can lower our estimate of the Alfvén wave total intensity. As to the wave spectrum in the short wavelength range, it should be well described by the quasi-linear theory unless the ion distribution over the large $\left(90^{\circ}\right)$ pitch-angles is very anisotropic.

The spectra of the Alfvén wave turbulence obtained during the ICE flyby near comet GiacobiniZinner and claimed to be of the Kolmogorov type (Tsurutani and Smith (1986a)) have raised doubts concerning the validity of the quasi-linear theory (Sagdeev et al. (1986)) and have stimulated attempts (Goldstein and Roberts (1987)) to construct a non-linear theory of the strong Alfvén wave turbulence. However, later analysis of the plasma and magnetic field (Glassmeier et al. (1987), (1989)) measurements from the Giotto spacecraft near comet Halley demonstrated that the slopes of the Alfvén wave turbulence spectra are certainly steeper than that of the Kolmogorov spectrum. This could be because the level of turbulence near comet Halley is lower than near comet GiacobiniZinner. Moreover, the spectral energy density of the magnetic field oscillations obtained for the solar wind flow nearly perpendicular to the magnetic field (Figure 4) shows that this spectrum could not be formed as a result of turbulence cascading towards the short scales. Indeed, if we assume that the linearly excited waves represent the energy source of turbulence, then this source is in the short waves $\left(f \approx f_{c i} / \cos \alpha\right.$ shaded area in Figure 4$)$ as calculations by Wu and Davidson (1972), 
Gary et al. (1984) and Winske et al. (1985) predict. Therefore, the wave cascade should form a spectrum with different slopes in the long wave and short wave ranges relative to the source region, contrary to the observations of a constant spectral slope, $B_{f}^{2} \propto f^{-2}$, predicted by the quasi-linear theory (Sagdeev et al. (1986), Galeev et al. (1987b)). Let us note that a small bump in the source area seen in Figure 4 is also described by Equation (25). Finally, we give the numerical estimate of the spectral energy density (Equation (25)) for typical solar wind and cometary gas parameters $\left(V_{g} \tau=2 \times 10^{6} \mathrm{~km}\right.$, Gringauz et al. (1986))

$$
\begin{aligned}
B_{f}^{2} & \simeq 10^{5}\left(\frac{V_{\mathrm{A}}}{u_{\infty}}\right)\left(\frac{2.5 \mathrm{mHz}}{f}\right)^{2}\left[1-\frac{B}{5 \mathrm{nT}}\left(\frac{2.5 \mathrm{mHz}}{f}\right)\right] \times \\
& \times\left(\frac{B}{5 \mathrm{nT}}\right)\left(\frac{Q}{10^{30} \mathrm{~s}^{-1}}\right)\left(\frac{10^{6} \mathrm{~km}}{r}\right)\left(\frac{u_{\infty}}{400 \mathrm{~km} / \mathrm{s}}\right) \frac{\mathrm{nT}^{2}}{\mathrm{~Hz}}
\end{aligned}
$$

This expression agrees well with the observations shown in Figure 4. However, the quasi-linear theory cannot describe the non-linear MHD waves observed near comet Giacobini-Zinner (Tsurutani and Smith (1986b), Tsurutani et al. (1987)). Some attempts to explain these observations have been undertaken using linear theory analysis (Brinca and Tsurutani (1987), Gary and Madland (1988), Goldstein and Wong (1987), Winske and Gary (1986)) and non-linear theory and computer simulations (Kennel et al. (1988), Omidi and Winske (1988), Gary et al. (1989)).

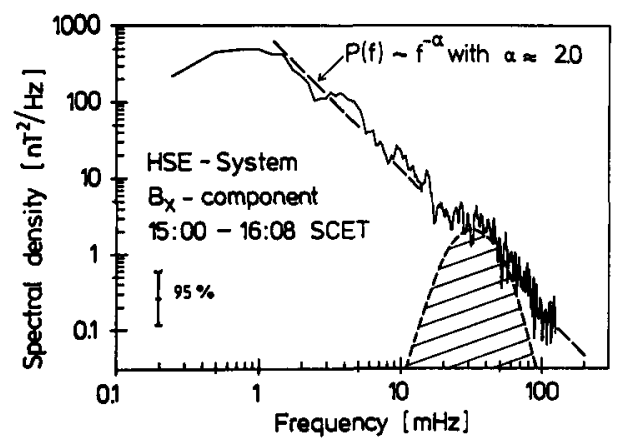

Figure 4. Power spectrum of the $B_{x}$ component in the region of solar wind flow quasi-perpendicular to the magnetic field vector. The hatched area marks the spectral range (from Glassmeier et al. (1989)).

\section{Solar Wind Flow Near Comets}

\subsection{SEMIKINETIC DESCRIPTION OF THE UNSHOCKED SOLAR WIND}

As we have shown in the previous section, the interaction of cometary ions with Alfvén waves results in the strong pitch-angle scattering without significant change in the ion energy. This means that the cometary ions born in the solar wind with energies much higher than the thermal energy of solar wind particles represent a separate component of plasma. In other words, solar wind plasma loaded by cometary ions remains unthermalized and one has to describe these two components 
separately (Wallis and Ong (1975), Galeev et al. (1985a)). If we neglect the small slipping of the cometary ions relative to the solar wind on the order of $V_{\mathrm{A}} / u \ll 1$, then the equation for the balance of mass and the equation of plasma motion can be written in the form of MHD equations

$$
\begin{gathered}
d(\rho u) / d x=m_{i} \dot{N} \\
d\left(\rho u^{2}\right) / d x=-d P / d x,
\end{gathered}
$$

where $\rho$ is the mass density of the loaded solar wind and $P$ is the plasma pressure. For the sake of simplicity, we will assume here that the main contribution to the plasma pressure comes from the cometary ions

$$
P=\left(4 \pi m_{i} / 3\right) \int_{0}^{\infty} v^{4} f_{0}(x, v) d v
$$

Therefore, for the closure of Equations (32)-(34), we must solve the kinetic equation (16) for the velocity distribution of cometary ions. Neglecting the small terms on the order of $V_{\mathrm{A}} / u$ in this equation, we rewrite it in the form

$$
u \frac{\partial f_{0}}{\partial x}-\frac{1}{3} \frac{d u}{d x} v \frac{\partial f_{0}}{\partial v}=\frac{\dot{N}}{4 \pi v^{2}} \delta(v-u)
$$

Using Equation (32) and the new variables $\left(u, \chi+v^{3} u\right)$ instead of the old ones $(x, v)$, we find the following solution of Equation (35) (Galeev et al. (1987b))

$$
f_{0}(u, \chi)=\frac{3}{4 \pi} \int_{u_{\infty}}^{u} \delta\left(\chi / u^{\prime}-u^{3}\right) \frac{d\left(\rho^{\prime} u^{\prime}\right)}{d u^{\prime}} d u^{\prime}
$$

Calculating the plasma pressure with the help of this solution, we can obtain the solution of Equation (33) of plasma motion that coincides with the well-known result usually derived from the MHD equations for the plasma with the specific heat ratio $\gamma=5 / 3$

$$
\rho u=\rho_{\infty} u_{\infty}\left(\frac{5 u_{\infty}}{4 u}-\frac{u_{\infty}^{2}}{4 u^{2}}\right)
$$

Thus we recover from Equations (33) and (37) the results of Bierman et al. (1967):

$$
\begin{gathered}
\frac{u}{u_{\infty}}=\frac{5}{8}\left(\frac{\rho_{\infty} u_{\infty}}{\rho u}\right)\left[1 \pm \sqrt{1-\frac{16}{25} \frac{\rho u}{\rho_{\infty} u_{\infty}}}\right] \\
\frac{P}{\rho_{\infty} u_{\infty}^{2}}=\frac{1}{8}\left[3 \mp 5 \sqrt{1-\frac{16}{25} \frac{\rho u}{\rho_{\infty} u_{\infty}}}\right]
\end{gathered}
$$

To describe the unshocked (shocked) solar wind flow, we should use the upper (lower) signs here. However, as was pointed by Bierman et al. (1967), there is no continuous transition from the supersonic to the subsonic flow, and according to the computer simulations (Schmidt and Wegmann (1982), Baranov et al. (1986), Wegmann et al. (1987)), the bow shock forms in front of the comet as soon as the local Mach number drops to the value $M=2$. Analysis of the perturbations of the stationary solar wind flow described by Equations (32)-(34) in its turn shows (Galeev and Khabibrakhmanov (1990)) that this flow is unstable for $M \leq 2$ and the instability takes form of the gradient catastrophe that could result in the shock formation. Using Equations (38) and (39), we find that value $M=2$ is reached when $\rho u / \rho_{\infty} u_{\infty}=700 / 529=1.32$.

The plasma velocity and pressure upstream and downstream of the shock are, respectively,

$$
u_{1} / u_{\infty}=0.657 ; \quad P_{1} / \rho_{\infty} u_{\infty}^{2}=0.13 ; \quad u_{2} / u_{\infty}=0.287 ; \quad P_{2} / \rho_{\infty} u_{\infty}^{2}=0.62
$$


The position of the shock is easily found from the solution of Equation (32) for the value of $\rho u$ given above and agrees well with the observations near comet Halley (Gringauz et al. (1986), Klimov et al. (1986), Johnstone et al. (1986), Balsiger et al. (1986), Reme et al. (1986), Mukai et al. (1986), Galeev et al. (1986a)). To compare these observations with our one-dimensional calculations, the shape of cometary bow shock was taken from the numerical simulation by Galeev and Lipatov (1984) (see also the discussion in Galeev (1987)).

Finally, let us note that, taking account of the finite proton, electron and magnetic field pressures as given by Equation (40), a cometary bow shock with $\mathbf{M}=2$ will stand further from the comet and the pressure of cometary ions at the shock will be lower.

\subsection{ACCELERATION OF COMETARY IONS NEAR COMETS}

During the encounter of the ICE spacecraft with comet Giacobini-Zinner, large fluxes of energetic cometary ions were registered in a huge area upstream of the comet filled by the Alfvén waves (Hynds et al. (1986)). It was expected that the energy of the picked-up cometary ions would be about one hundred times higher than the kinetic energy of solar wind protons. However, this observations, as well as measurements near comet Halley (Somogyi et al. (1986), McKenna-Lawlor et al. (1986)), showed that ions with energies up to $0.5 \mathrm{MeV}$ had appreciable fluxes. The first suggestion of possible acceleration of cometary ions was made by Amata and Formisano (1985), who envisaged that diffusive shock acceleration (see the review by Forman and Webb (1985))) could be very effective in the turbulent cometary plasma environment. There is no question that this mechanism works near the cometary bow shock and could be well-described theoretically (see the review by Ip and Axford (1989)). However, far from the bow shock, where the MHD turbulence is generated by the cometary ion pickup processes and the energetic ions are born, the stochastic (or second-order Fermi) acceleration process becomes more effective than the diffusive shock acceleration. The measurements by Hynds et al. (1986), Coates et al. (1986b), and Wilken et al. (1986) could be fitted well by an exponential velocity distribution of cometary ions (Figure 5) which also witnesses in favor of the stochastic acceleration. Ip and Axford (1986) discussed this acceleration in terms of a Fokker-Planck equation for the differential number density with model coefficients for energy diffusion and pitchangle scattering. Gribov et al. (1986) have made an attempt to describe the energy diffusion by the quasi-linear velocity diffusion coefficient with the spectral energy density of magnetic field oscillations calculated from the data measured aboard the VEGA-1 spacecraft (see also Barbosa (1989)). We derive here the kinetic equation for accelerated cometary ions by averaging the quasi-linear collision term (Equation (9)) over pitch angles, with the help of Equation (20) for the anisotropic part of the ion distribution function. As a result, we obtain (Skilling (1975), Forman and Webb (1985)):

$$
\begin{aligned}
u \frac{\partial f_{0}}{\partial x} & -\frac{1}{3} \frac{d u}{d x} v \frac{\partial f_{0}}{\partial v}=\frac{1}{v^{2}} \frac{\partial}{\partial v} v^{2} D_{v v} \frac{\partial f_{0}}{\partial v}+ \\
& +\frac{1}{8} \int_{-1}^{+1} d \mu\left(1-\mu^{2}\right)\left\{\frac{\partial}{\partial x} v \cos \alpha-\frac{1}{v^{2}} \frac{\partial}{\partial v} v^{2}\left[u \cos \alpha-\mu v\left(\sin ^{2} \alpha-\frac{2}{3}\right)\right] \frac{d u}{d x}\right\} \times \\
& \times \frac{1}{\nu_{\mu \mu}}\left\{v \cos \alpha \frac{\partial f_{0}}{\partial x}-\left[u \cos \alpha-\mu v\left(\sin ^{2} \alpha-\frac{2}{3}\right)\right] \frac{d u}{d x} \frac{\partial f_{0}}{\partial v}\right\},
\end{aligned}
$$

where the energy diffusion coefficient

$$
D_{v v}=\frac{1}{2} \int_{-1}^{1}\left(1-\mu^{2}\right)\left[\nu_{v v}-\frac{\nu_{v \mu} \nu_{\mu v}}{\nu_{\mu \mu}}\right] d \mu
$$

is expressed through the collision frequencies $\nu_{\alpha \beta}$ given by Equation (10). The second term on the right-hand side describes both spatial diffusion with the coefficient (Jokipii (1971))

$$
D_{x x}=\frac{v^{2}}{8} \int_{-1}^{1} d \mu\left(1-\mu^{2}\right) / \nu_{\mu \mu}
$$


11 September $1985,1002-1013$ UT

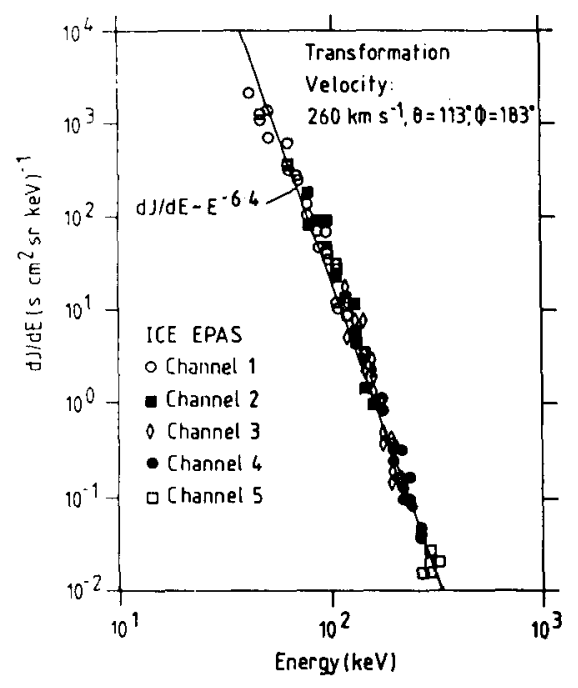

11 September 1985, 1002-1013 UT

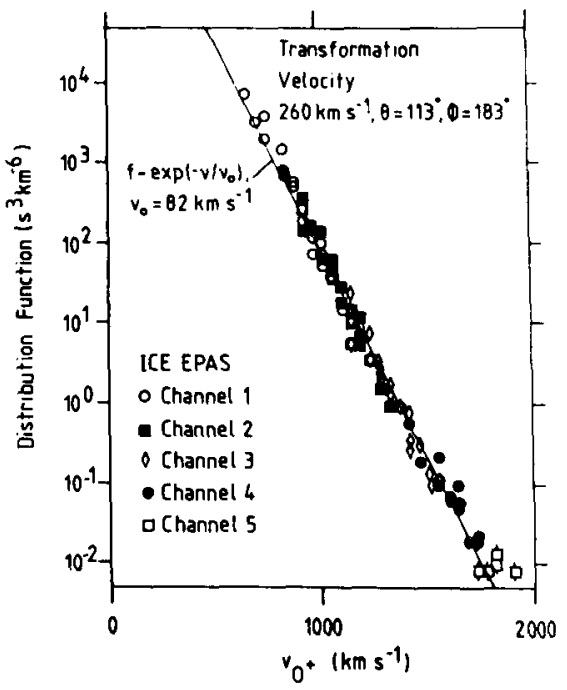

Figure 5. The energy and velocity distributions of cometary ions approximated by power law and exponential distributions, respectively (from Hynds et al. (1986)).

and viscous heating. However, for the energetic $(v \gg u)$ accelerated ions, viscosity is smaller than spatial diffusion by the factor $(u / v)^{2}$ and we neglect it.

Let us note here that the energy diffusion coefficient turns out to be zero if the particle with a given velocity interacts with Alfvén waves propagating only in one particular direction, as it is true in our case (Figure 2). This becomes evident if we rewrite Equation (42) in the form

$$
D_{v v}=2 \int_{-1}^{1}\left(1-\mu^{2}\right) \frac{\nu_{+} \nu_{-}}{\nu_{+}+\nu_{-}} V_{\mathrm{A}}^{2} d \mu
$$

where $\nu_{+}$and $\nu_{-}$are the frequencies of pitch-angle scattering by Alfvén waves propagating in the positive and negative directions along the magnetic field. In other words, the Fermi acceleration in general requires the presence of oppositely propagating waves (or magnetized clouds), and therefore there is no stochastic acceleration in our quasi-linear theory of solar wind loading by cometary ions. However, if we take into account the fact that Alfven waves of both polarizations propagate from the solar corona into interplanetary space, i.e., antisunward, then we find that these waves and those excited by the cometary ions result in energy diffusion of cometary ions with parallel velocity in the range $-u \cos \alpha<v_{\|}<u$ (see Figure 2). However, using Equation (25) for the spectrum of Alfvén waves near comets to calculate the pitch-angle scattering frequency from Equation (10), we find that the high-energy ions $(v \gg u)$ are scattered by these waves only in a small area of the velocity space $(|\mu|<u / v)$. The energy diffusion coefficient calculated from Equation (44) also scales as this small parameter. Using this calculated energy diffusion coefficient, we can solve Equation (42) and find that the velocity distribution of accelerated ions falls exponentially, with the exponent approximately proportional to the cube of velocity. 


\section{Structure Of The Cometary Bow Shock}

Strong pitch-angle scattering of cometary ions by excited Alfvén waves justifies an MHD description of the solar wind flow loaded by cometary ions as the flow of a gas with the specific heat ratio $\gamma=5 / 3$. In this approximation, the position of the cometary bow shock could be found quite accurately and even the bulk parameters of the plasma (treated as a single fluid) on both sides of a shock could be calculated. However, to describe the structure of the cometary bow shock, one needs to consider the solar wind plasma and the picked-up cometary ions as two different components of plasma, since the internal spatial scales of these two components (essentially the cyclotron radii of ions) differ by an order of magnitude. Moreover, these two components are not in thermal equilibrium, as we have already noted. As a possible compromise, we have described the solar wind loaded by cometary ions through treating the cometary ions as a hot gas contributing mainly to the internal energy of the plasma and to a smaller extent to the bulk kinetic energy and the solar wind as a single fluid. This compromise is no longer possible when we consider the cometary bow shock structure, since cometary ions and solar wind protons behave very differently across the shock. Therefore, hybrid numerical codes were used to study the structure of the shock (Galeev et al. (1985b), Omidi and Winske (1987), Lipatov and Syrovatskii (1987)).

\subsection{RESULTS OF COMPUTER SIMULATIONS}

The first computer simulation of the cometary bow shock was done by Galeev et al. (1985b) for the simplest case of a shock propagating perpendicular to the ambient magnetic field. In this case, the pickup of cometary ions takes place through their $E \times B$ drift, where $E$ and $B$ are the macroscopic electric and magnetic fields in the solar wind as viewed in the comet's rest frame. In other words, the magnetic field lines couple the two above specified components of the plasma and there is no need to consider the details of the collective coupling of the plasma components through the Alfvén waves, as is necessary for oblique or parallel shock propagation. This greatly simplifies the computations and also permits use of the one-dimensional hybrid code that describes the electrons as a massless fluid and treats the protons and cometary ions as macroparticles. The only disadvantage of this approach is that the velocity space of protons and ions is two-dimensional, i.e., the ion gas has the specific heat ratio $\gamma=2$ and thus wave excitation and pitch angle scattering are ignored.

The results of this simulation are shown in Figure 6 . The profiles of the plasma mass density $\rho$, the mass density of cometary ions $\rho_{i}$, and the magnetic field strength $B$ normalized to their upstream values are plotted in the upper part of the figure. The distribution of cometary ions and plasma protons in velocity space (the $v_{x}, v_{y}$ plane is perpendicular to $\mathbf{B}$ ) is displayed in the lower part of the figure. The spatial coordinate $z$ is given in units of the cyclotron radius of cometary ions, which were assumed to have a ring distribution upstream of the shock, with speeds in the range of $(1 \div 1.5) u_{\infty}$ and a mass $m_{i}=5 m_{p}$. The mass density of cometary ions was about $30 \%$ that of the protons, the Alfvén Mach number $M_{A}=8$, and the typical values of the upstream plasma betas were $\beta_{e}=1$ and $\beta_{p}=0.5$. Both protons and ions enter the computation area at the left boundary $(z=0)$ and leave it at the right boundary $(z=10)$. We see that the cometary ions, due to their large velocity of cyclotron motion, easily leak forward from behind the shock front and are turned back by the Lorentz force. The incoming solar wind, in turn, is decelerated by the cometary ions. As a result, the plasma is compressed and the magnetic field enhanced at the foot of the shock in a region roughly the size of the cyclotron radius of the cometary ions. The subshock is formed at the end of the foot, and the magnetic field and plasma density overshoot behind it. Let us note that cometary ions do not change their cyclotron velocity very much across the subshock. Also, the cometary ions leaking forward are accelerated in the foot by the self-consistent electric field of the plasma flow, and thus a beam of ions bunched in the phase of their cyclotron rotation is clearly seen in the lower part of Figure 6. In contrast, the solar wind protons are heated while they cross the subshock and some of them are reflected from the subshock and accelerated in the foot. Thus the 
cometary bow shock can be described as a shock with an isothermal jump across which the cometary ions are almost isothermal and the subshock itself is a supercritical ( $M \geq 3)$ proton shock. In the next subsection, we calculate the bulk parameters of the two plasma components on both sides of a shock using this assumption.
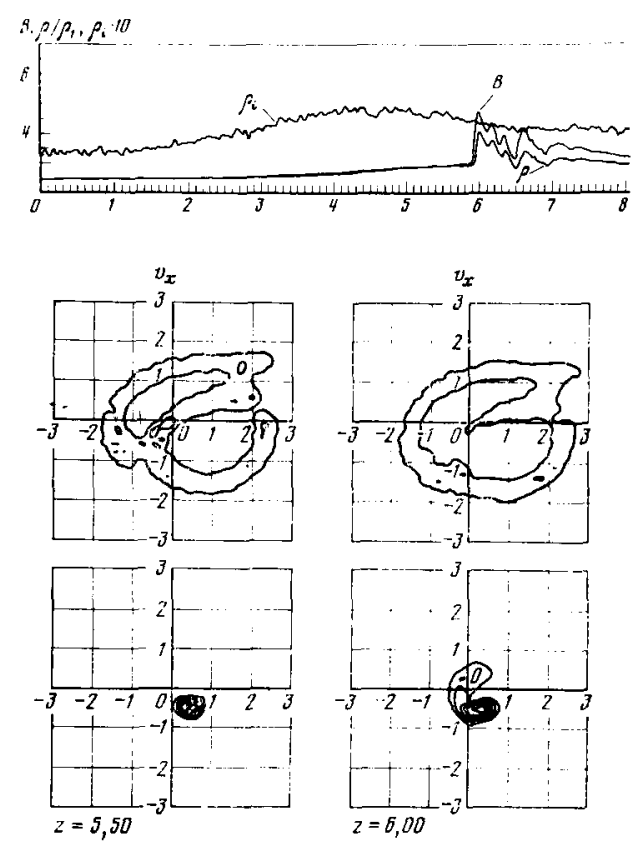

Figure 6. The profiles of plasma mass density $\rho$, cometary ion mass density $\rho_{i}$, magnetic field strength $B$ (top panel), and the distribution of cometary ions and protons in the shock front obtained by numerical simulations (from Galeev et al. (1985b)).

Omidi and Winske (1987) have undertaken a more advanced computer simulation that addresses the effect of mass loading on the cometary shock formation and structure. For this they have used the electromagnetic hybrid simulation code. The length of the system was $1500 c / \omega_{p i}\left(\omega_{p i}\right.$ is the proton plasma frequency) and the production rate of cometary ions was modelled by allowing newborn singly charged oxygen ions to vary through the length of the system to model the solar wind flow at radial distances of $10^{5}<r<2 \times 10^{5} \mathrm{~km}$ from the nucleus of a comet with a natural production rate of $10^{30} \mathrm{~mol} / \mathrm{s}$. Another purpose of this simulation was to study the shock structure for different angles of propagation. Both solar wind protons and electrons were assumed to have $\beta=1$ and the Alfvén Mach number was $M_{A}=6$. This simulation confirmed all the results of Galeev et al. (1985b) for the quasi-perpendicular cometary shock. The structure of an oblique shock $\left(\theta=50^{\circ}\right.$ is the angle between the shock normal and $B$ ) was found to be completely different. The solar wind and cometary ion densities, as well as the magnetic field strength, gradually increased across a very diffuse (more than five oxygen ion cyclotron radii) shock front where large-amplitude electromagnetic waves were present. These waves resulted in both the deceleration and heating of the protons. Since there was no subshock found in this case, we can assume that heat conductivity alone could maintain the shock 
transition. One would expect that this would be true for the quasi-parallel shock as well. But quite unexpectedly, Omidi and Winske (1987) discovered that the quasi-parallel shock transition $\left(\theta=5^{\circ}\right)$ finally evolves into a proton subshock with an extended foot most clearly seen in the magnetic field profile (Figure 7). As in the case of the oblique shock, large-amplitude electromagnetic waves are excited by the cometary ions moving relative to the solar wind, thus providing the coupling of the two plasma components. However, in contrast to the oblique shock, the fluctuations of the proton and ion densities and the magnetic field magnitude are highly uncorrelated. A strong heating of protons takes place at the subshock and downstream of it. The formation of an intense subshock in this case could be the result of nonlinear steepening of excited waves, demonstrated both by the hybrid simulation (Omidi and Winske (1988)) and by the numerical analysis of nonlinear wave equations (Malkov et al. (1989)). As was shown in these papers, the nonlinear wave steepening is responsible for the formation of shocklets which are convected downstream as observed during the ICE encounter with comet Giacobini-Zinner (Tsurutani and Smith (1986b), Tsurutani et al. (1987)).

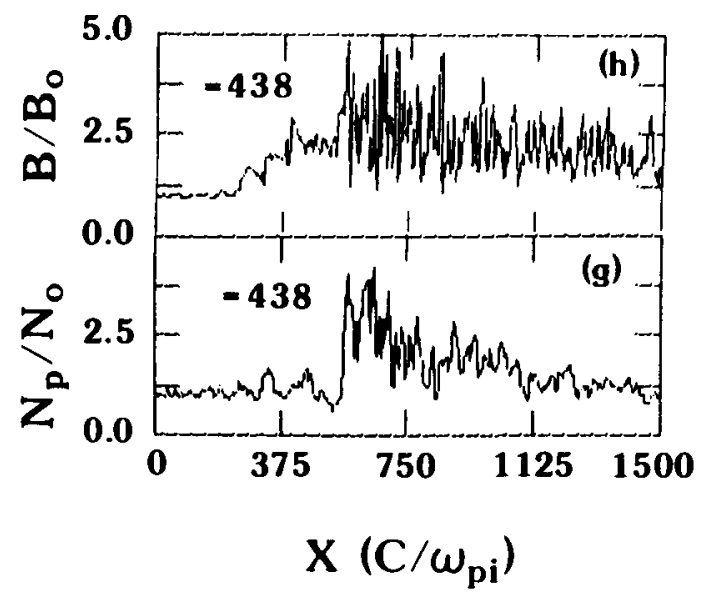

Figure 7. The profiles of the proton density and magnetic field in the quasi-parallel cometary shock (from Omidi and Winske (1987)).

\subsection{SUBSHOCKS IN COMETARY BOW SHOCKS}

Viscous subshocks in thermal conductive shocks are described by the mass, momentum and energy conservation equations in the form (Landau and Lifshitz, 1986):

$$
\begin{gathered}
\rho u \equiv j=\text { const } \\
P+j u=P_{1}+j u_{1}+\rho \nu d u / d x \\
\frac{\gamma p u}{\gamma-1}+j u^{2} / 2=\frac{\gamma P_{1} u_{1}}{\gamma-1}+j u_{1}^{2} / 2+\kappa \frac{d T}{d x}
\end{gathered}
$$


where the $x$ axis is directed downstream perpendicular to the shock front, $\nu$ is the viscosity, $\kappa$ the thermal conductivity, $\gamma$ the specific heat ratio, and $T$ the plasma temperature. The indexes 1 and 2 refer to the upstream and downstream plasma parameters. The flow velocity and pressure of plasma downstream of the shock are found from these equations, assuming plasma uniformity there, as

$$
\begin{aligned}
\frac{u_{2}}{u_{1}} & =\frac{1}{\gamma+1}\left[\gamma-1+\frac{2}{M_{1}^{2}}\right] \\
-\frac{P_{2}}{P_{1}} & =\frac{1}{\gamma+1}\left[2 \gamma M_{1}^{2}-\gamma+1\right],
\end{aligned}
$$

where $M_{1}$ is the upstream Mach number calculated in a single fluid model.

First we consider the subshock in the quasi-perpendicular cometary shock, where both the cometary ion temperature and pressure are continuous across the subshock. In this case, the continuous plasma pressure buildup while plasma is decelerated in the shock transition is not possible under the condition

$$
\left.\frac{\partial P_{i}}{\partial u}\right|_{u=u_{2}}>0
$$

Assuming that the proton pressure increases adiabatically in the foot of a shock controlled by the cometary ion heat conduction (small obliqueness is assumed here also) and using Equation (47) in the limit $\nu \rightarrow 0$ to obtain $P_{i}$ as a function of the velocity $u$, we rewrite this condition in the well-known form

$$
\gamma P_{g 2} / j u_{2}>1
$$

where $p_{g}$ is the gas pressure of the electron-proton solar wind plasma. This condition was used by Galeev et al. (1985b) to find the critical gas pressure for the subshock to form in the quasiperpendicular cometary bow shock:

$$
\frac{P_{g 1}}{P_{i 1}+P_{g 1}}>M_{1}^{2}\left[\frac{2+(\gamma-1) M_{1}^{2}}{(\gamma+1) M_{1}^{2}}\right]^{\gamma+1}
$$

It was shown that for the typical plasma parameters near the comet used in simulations, this condition is not satisfied. However, in a collisionless plasma, quasi-perpendicular shocks have overshoots behind the ramps where the plasma velocity $u_{2 *}$ drops below the Hugoniot value $u^{2}\left(u_{2 *}=0.5 u_{2}\right)$, so that Equations (52) and (53) are fulfilled and a subshock is formed (Galeev et al. (1985b)). Plasma parameters just prior to this subshock are found from Equation (47), assuming the ion density, temperature and pressure are continuous across the subshock. Assuming also that the proton pressure increases across the weak subshock approximately adiabatically, we rewrite Equation (47) in the form

$$
P_{g 1}\left(\frac{u_{1}}{u_{2 *}}\right)^{\gamma}\left[1-\left(\frac{u_{2 *}}{u^{\prime}}\right)^{\gamma}\right]=j u^{\prime}\left[1-\frac{u_{2 *}}{u^{\prime}}\right]
$$

where $u^{\prime}$ is the plasma flow velocity prior the subshock. In the case of $\gamma=2$, this equation has the simple solution

$$
\frac{u^{\prime}}{u_{2 *}}=\frac{P_{g 2 *}}{2 j u_{2 *}}\left[1+\sqrt{1+\frac{4 j u_{2 *}}{P_{g 2 *}}}\right]
$$

From the requirement $u^{\prime}>u_{2 *}$, we recover Equation (52). To find the numerical value of parameters, one should use here the overshoot values for $P_{g 2}$ and $u_{2}$.

Since there is no overshoot in the oblique shocks, we cannot expect a subshock in an oblique cometary bow shock, in agreement with the computer simulation of Omidi and Winske (1987). 


\subsection{OBSERVATIONS OF COMETARY BOW SHOCK}

While plasma (Bame et al. (1986)) and magnetic field (Smith et al. (1986)) observations near comet Giacobini-Zinner could not identify the bow shock on the background of the large plasma and magnetic field fluctuations in a turbulent solar wind, the plasma wave measurements (Coroniti et al. (1986), Scarf et al. (1986), Scarf (1989)) provided electric and magnetic field spectra that are characteristic of a shock wave crossing. The spectral amplitudes of the electric and magnetic field oscillations are displayed on the top and bottom panels of Figure 8, respectively. Similar to the quasi-perpendicular Earth bow shock, the electron plasma oscillations (Langmuir waves) are generated just upstream of the cometary shock transition at a distance of $\sim 1.5 \times 10^{5} \mathrm{~km}$ from the comet. Across this transition, the intensities of both ion acoustic and whistler waves jump and continue to increase throughout the diffuse bow shock. Though these waves are characteristic of the quasi-perpendicular Earth bow shock, their intensity near the comet was unexpectedly high. The intense whistler waves are closely associated with the electron heat flux from the downstream plasma (Fuselier et al. (1986)) and thus could be responsible for the generation of whistlers. The beams of newly created cometary ions could contribute to the generation of the ion acoustic waves (Kennel et al. (1986)). The diffuse character of the Giacobini-Zinner bow shock was interpreted to be characteristic of an oblique cometary shock (Omidi and Winske (1987)). However, the initial discontinuity in the measured intensities and spectra of plasma waves has a width on the order of the water ion cyclotron radius and thus should be interpreted as the quasi-perpendicular cometary bow shock.

The presence of a bow shock near comet Halley was clearly identified by plasma, magnetic field and plasma wave measurements aboard the VEGA-1 and VEGA-2 spacecraft (Gringauz et al. (1986), Riedler et al. (1986), Klimov et al. (1986), Galeev et al. (1986)). Because of the greater time resolution of the plasma wave analyser, it was used to estimate the width of the quasi-perpendicular bow shock crossed by the VEGA-1 spacecraft. Here the intensity of the lowerhybrid waves jumped in about 12 seconds, which corresponds to the shock width of the order of water group ion cyclotron radius (Klimov et al. (1986)). However, since the time resolution of the plasma analyser was too low to describe the fine structure of the bow shock, we use here the data of measurements aboard the Giotto spacecraft to illustrate the complex structure of the cometary bow shock. These data (Johnstone et al. (1986), Reme et al. (1986)) were analyzed by Coates et al. (1987) and are displayed in Figure 9. We see that the electron density and the cometary ion density increased through the relatively broad $(36,000 \mathrm{~km}$, from $\approx 19: 20$ to $19: 31 \mathrm{SET})$ shock transition. It is important to note that the solar wind deceleration at the shock was not monotonic and had three abrupt changes of the velocity and magnetic field vectors (see the detailed discussion by Neugebauer et al. (1989b)). Analysis of these data identifies two different characteristic scales. The cometary bow shock transition and the cometary ion heating take place at the scale of the scale of the cometary ion cyclotron radius (Coates et al. (1987), Neugebauer et al. (1989b)). But the solar wind velocity jumps at the end of this transition on the spatial scale on the order of $500 \mathrm{~km}$, which is comparable to the solar wind proton cyclotron radius (Coates et al. (1987)). So we can conclude that a subshock is formed within the quasi-perpendicular cometary bow shock in this case. The solar wind parameters upstream of this shock $\left(n_{p}=n_{e}=7 \mathrm{~cm}^{-3}, B=7 \mathrm{nT}, v_{T p}=68 \mathrm{~km} / \mathrm{s}\right.$, $T_{e}=2.8 \cdot 10^{5} \mathrm{~K}, n_{i}=0.03 \mathrm{~cm}^{-3}, u_{1}=300 \mathrm{~km} / \mathrm{s}$ ) given by Neugebauer et al. (1988) permit us to check the criterion (Equation (53)) for the subshock formation. Since the total gas pressure (i.e., solar wind electrons, protons and magnetic field) is about equal to the cometary ion pressure, this criterion is satisfied for the Mach number $M=2$ shock. The assumption that this shock is quasiperpendicular does not contradict the above data for the nominal shock normal and magnetic field orientations. It is interesting to note also that the plasma pressure increases across the shock by a factor of approximately two, i.e., this increase is smaller than that calculated in the one-fluid model. This is consistent with the subshock formation. 

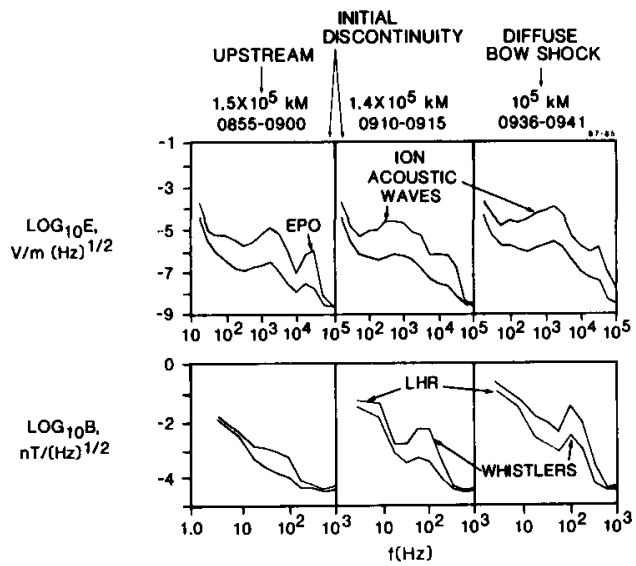

Figure 8. Characteristic peak and average E and B wave spectra measured as ICE moved through the comet Giacobini-Zinner bow shock (from Scarf et al. (1987)).

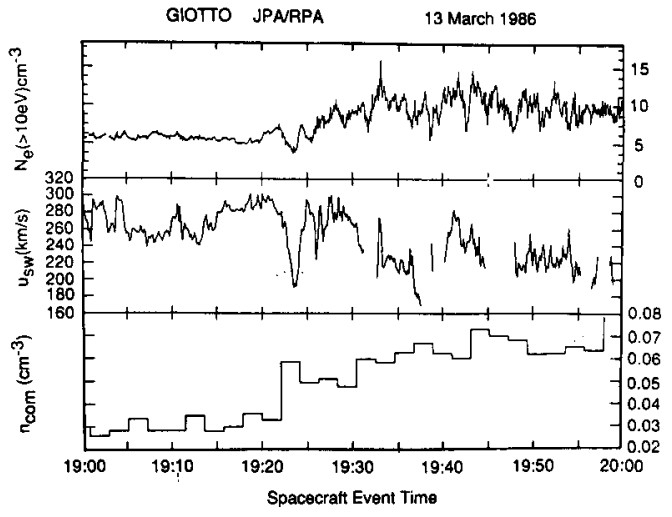

Figure 9. Electron density, solar wind proton speed and cometary ion density profiles as Giotto moved through the comet Halley bow shock (from Coates et al. (1987)). 


\section{Conclusion}

The encounters of spacecraft with comets revealed a variety of new plasma phenomena accompanying the solar wind interaction with comets. The majority of these phenomena, i.e., MHD solar wind turbulence, solar wind loading by cometary ions, and specific cometary bow shocks, are understood and described in general by the tools of modern plasma physics. However, the details of all of these phenomena need further study.

\section{References}

Amata, E., and Formisano, V. (1985) 'Energization of positive ions in the cometary foreshock region,' Planet. Space Sci. 33, 1243-1250.

Balsiger, H., Altwegg, K., Buhler, F., Feiss, J., Ghielmetti, A.G., Goldstein, B.E., Goldstein, R., Huntress, W.T., Ip, W.-H., Lazarus, A.J., Meier, A., Neugebauer, M., Rettenmund, U., Rosenbauer, H., Schwenn, R., Sharp, R.D., Shelley, E.G., Ungstrup, E., and Young, D.T. (1986). 'Ion composition and dynamics at comet Halley,' Nature 321, 330-335.

Bame, S.J., Anderson, R.C., Asbridge, J.R., Baker, D.N., Feldman, W.C., Fuselier, S.A., Gosling, J.T., McComas, D.J., Thomsen, M.F., Young, D.T., and Zwickl, R.D. (1986) 'Comet GiacobiniZinner: Plasma description,' Science 232, 356-361.

Baranov, V.B., Zaitsev, N.A., and Lebedev, M.G. (1986) 'Numerical simulation of solar wind interaction with comets,' Astron. Zurn. 63, 170.

Barbosa, D.D. (1989) 'Stochastic acceleration of cometary pickup ions: The classic leaky box model,' Astrophys. J. 341, 493-503.

Bell, A.R. (1978) 'The acceleration of cosmic rays in shock-fronts, I.' Mon. Not. R. Astron. Soc. $182,147-156$.

Bierman, L., Brosowski, B., and Schmidt, H.U. (1967) 'The interaction of the solar wind with a comet,' Solar Phys. 1, 254-283.

Brinca, A.L., and Tsurutani, B.T. (1987) 'On the polarization, compression and nonoscillatory behavior of hydromagnetic waves associated with pickup ions,' Geophys. Res. Lett. 14, 495498.

Coates, A.J., Johnstone, A.D., Wilken, B., Jockers, K., and Glassmaier, K.-H. (1989a) 'Velocityspace diffusion of pickup ions from the water-group at comet Halley,' J. Geophys., Res., in press.

Coates, A.J., Johnstone, A.D., Wilken, B., Jockers, K., and Glassmaier, K.-H. (1989b) 'Observations of heavy pick-up ions at Halley: Pitch angle and energy distributions,' J. Geophys. Res., submitted.

Coates, A.J., Lin, R.P., Wilken, B., Amata, E., Anderson, K.A., Borg, H., Bryant, D.A., Carlson, C.W., Curtis, D.W., Formisano, V., Jockers, K., Johnstone, A.D., Korth A., Mendis, D.A., Reme, H., Richter, A.K., Rosenbauer, H., Sauvaud, J.A., Studeman W., Thomsen, M.F., d'Uston, C., and Winningham, J.D. (1987) 'Giotto measurements of cometary and solar wind plasma at the comet Halley bow shock,' Nature 327, 489-490.

Coroniti, F.V., Kennel, C.F., Scarf, F.L., Smith, E.J., Tsurutani, B.T., Bame, S.J., Thomsen, M.F., Hynds, R., and Wenzel, K.P. (1986) 'Plasma wave turbulence in the strong coupling region at comet Giacobini-Zinner,' Geophys. Res. Lett. 13, 869-872.

Forman, M.A., and Webb, G.M. (1985) 'Acceleration of energetic particles,' in R.A. Stone and B.T. 'Tsurutani (eds.), Collisionless Shocks in the Heliosphere: A Tutorial Review, AGU, Washington, D.C., pp. 91-114.

Fuselier, S.A., Feldman, W.C., Bame, S.J., Smith, E.J., and Scarf, F.L. (1986) 'Heat flux observations and the location of the transition region boundary of Giacobini-Zinner,' Geophys. Res. Lett. $13,247-250$. 
Galeev, A.A. (1987) 'Encounters with comets: Discoveries and puzzles in cometary plasma physics,' Astron. Astrophys. 187, 12-20.

Galeev, A.A., and Khabibrakhmanov, I.Kh. (1990) 'On the Mach number of a cometary bow shock,' Pis'ma Astron. Zurn., in press (in Russian).

Galeev, A.A., and Lipatov, A.S. (1984) 'Plasma processes in the cometary atmospheres,' Adv. Space Res. 4, No.9, 229-237.

Galeev, A.A., and Sagdeev, R.Z. (1988) 'Alfven waves in a space plasma and its role on the solar wind interaction with comets,' Astrophys. Space Sci. 144, 427-438.

Galeev, A.A., Cravens, T.E., and Gombosi, T.I. (1985a) 'Solar wind stagnation near comets,' Astrophys. J. 289, 807-819.

Galeev, A.A., Gribov, B.E., Gombosi, T., Gringauz, K.I., Klimov, S.I., Olerz, P., Remizov, A.P., Riedler, W., Sagdeev, R.Z., Savin, S.P., Sokolov, A.Yu., Shapiro, V.D., Shevchenko, V.I., Szego, K., Verigin, M.I., and Yeroshenko, Ye.G. (1986) 'Position and structure of the comet Halley bow shock: Vega-1 and Vega-2 measurements,' Geophys. Res. Lett. 13, 841-844.

Galeev, A.A., Lipatov, A.S., and Sagdeev, R.Z. (1985b) 'Numerical simulation of near-comet shock waves: Some features of the structure and mechanisms of energy dissipation,' Sov. Phys. JETP $62,866-875$.

Galeev, A.A., Lipatov, A.S., and Sagdeev, R.Z. (1987a) 'Two-dimensional numerical simulation of the dynamics of cometary ion relaxation and MHD turbulence in the solar wind - cometary atmosphere interaction,' Sov. Phys. Plasma Phys. 13, 567-574.

Galeev, A.A., Polyudov, A.N., Sagdeev, R.Z., Shapiro, V.D., and Shevchenko V.I. (1987b) 'MHD turbulence caused by a comet in the solar wind,' Sov. Phys. JETP 65, 1178-1186.

Gary, S.P., and Madland, C.D. (1988) 'Electromagnetic ion instabilities in a cometary environment,' J. Geophys. Res. 93, 235-241.

Gary, S.P., Akomoto, K., and Winske, D. (1989) 'Computer simulation of cometary pick-up-ion/ion instabilities and wave growth,' J. Geophys. Res. 94, 3413-3525.

Gary, S.P., Smith, C.W., Lee, M.A., Goldstein, M.L., and Forslund, D.W. (1984) 'Electromagnetic ion beam instabilities,' Phys. Fluids 27, 1852-1862.

Glassmeier, K.H., Coates, A.J., Acuna, M.H., Goldstein, M.L., Johnstone, A.D., Neubauer, F.M., and Reme, H. (1989) 'Spectral characteristics of low-frequency plasma turbulence upstream of comet P/Halley,' J. Geophys. Res. 94, 37-48.

Glassmeier, K.H., Neubauer, F.M., Acuna, M.H., and Mariani, F. (1987) 'Low frequency magnetic field fluctuations in comet P/Halley's magnetosheath: Giotto observations,' Astron. Astrophys. $187,65-68$.

Goldstein, M.L., and Roberts, D.A. (1987) 'Numerical simulation of the generation of turbulence from cometary ion pick-up,' Geophys. Res. Lett. 14, 860-863.

Goldstein, M.L., and Wong, H.K. (1987) 'A theory for low-frequency waves observed at comet Giacobini-Zinner,' J. Geophys. Res. 92, 4692-4700.

Gombosi, T. (1988) 'Preshock region acceleration of implanted cometary $\mathrm{H}^{+}$and $\mathrm{O}^{+}$,' J. Geophys. Res. 93, 35-47.

Gribov, B.E., Keczkemety, K., Sagdeev, R.Z., Shapiro, V.D., Shevchenko, V.I., Somogyi, A.J., Szego, K., Erdo, S.G., Eroshenko, E.G., Gringauz, K.I., Keppler, E., Marsden, R., Remizov, A.P., Richter, A.K., Riedler, W., Schwingenshuh, K., and Wenzel, K.P. (1986) 'Stochastic Fermi acceleration of ions in the pre-shock region of comet Halley,' in Proc. 20th ESLAB Symp. on the Exploration of Halley's Comet, ESA SP-250, 271-276.

Gringauz, K.I., Gombosi, T.I., Remizov, A.P., Apathy, I., Szemerey, I., Verigin, M.I., Denchikova, L.I., Dyachkov, A.V., Keppler, E., Klimenko, I.N., Richter, A.K., Somogyi, A.J., Szego, K., Szendro, S., T'atrallyay, M., Varga, A., and Vladimirova, G.A. (1986) 'First in situ plasma and neutral gas measurements at comet Halley,' Nature 321, 282-285.

Hartle, R.E., and Wu, C.S. (1973) 'Effects of electrostatic instabilities on planetary and interstellar ions in the solar wind,' J. Geophys. Res. $78,5802-5807$. 
Hynds, R.J., Cowley, S.W.H., Sanderson, T.R., Wentzel, K.P., and Van Rooijen, J.J. (1986) 'Observations of energetic ions from comet Giacobini-Zinner,' Science 232, 361-365.

Ip, W.-H. (1980) 'Cometary atmospheres,' Astron. Astrophys. 92, 95-100.

Ip, W.-H., and Axford, W.I. (1982) 'Theories of physical processes in the cometary comae and ion tail,' in L.L. Wilkening (ed.), Comets, University of Arizona Press, Tucson, pp. 588-634.

Ip, W.-H., and Axford, W.I. (1986) 'The acceleration of particles in the vicinity of comets,' Planet. Space Sci. 34, 1061-1065.

Ip, W.-H., and Axford, W.I. (1987) 'A numerical simulation of charged particle acceleration and pitch-angle scattering in the turbulent plasma environment of comet Halley,' Proc. 20th Intern. Cosmic Ray Conf. (Moscow), Vol. 3, SH 4.2-14, p. 233.

Ip, W.-H., and Axford, W.I. (1989) 'Cometary plasma physics,' in W.F. Huebner (ed.), Physics of Comets in the Space Age, in press.

Isenberg, P.A. (1987) 'Energy diffusion of pickup ions upstream of comets,' J. Geophys. Res. 92, 8795-8799.

Johnstone, A.D., Coates, A.J., Heath, J., Thomsen, M.F., Wilken, B., Jockers, K., Formisano, V., Amata, E., Wonningham, J.D., Borg, H., and Bryant, D.A. (1987) 'Alfvenic turbulence in the solar wind flow during the approach to comet P/Halley,' Astron. Astrophys. 187, 25-32.

Johnstone, A., Coates, A., Kellock, S., Wilken, B., Jockers, K., Rosenbauer, H., Studemann, W., Weiss, W., Formisano, V., Amata, E., Cerulli-Irelli, R., Dobrowolny, M., Terenzi, R., Egidi, A., Borg, H., Hultqvist, B., Winningham, J., Gurgiolo, C., Bryant, D., Edwards, T., Feldman, W., Thomsen, M., Wallis, M.K., Bierman, L., Schmidt, H.U., Lust, Rh., Haerendel, G., and Paschmann, G. (1986) 'Ion flow at comet Halley,' Nature 321, 344-347.

Kennel, C.F., Coroniti, F.V., Scarf, F.L., Tsurutani, B.T., Smith, E.J., Bame, S.J., and Gosling, J.T. (1986) 'Plasma waves in the shock interaction regions at comet Giacobini-Zinner,' Geophys. Res. Lett. 13, 921-924.

Kennel, C.F., Mal'kov, M.A., Sagdeev, R.Z., Shapiro, V.D., and Khrabrov, A.V. (1988) 'Shockpacket of Alfven waves with dispersion,' Sov. Phys. JEPT Lett. 48, 75-78.

Klimov, S., Savin, S., Aleksevich, Ya., Avanesova, G., Balebanov, V., Balikhin, M., Galeev, A.A., Gribov, B., Nozdrachov, M., Smirnov, V., Sokolov, A., Vaisberg, O., Oberc, P., Krawczyk, Z., Crzedzielskii, S., Juchniewicz, J., Nowak, K., Orlowski, D., Parfianovich, B., Wozniak, D., Zbyszynski, Z., Voita, Ya., and Triska, P. (1986) 'Extremely-low frequency plasma waves in the environment of comet Halley,' Nature 321, 292-294.

Kulsrud, R.M. (1983) 'MHD description of plasma,' in A.A. Galeev and R.N. Sudan (eds.), Basic Plasma Physics I, North-Holland Publ. Co., Amsterdam New York Oxford, pp. 115-145.

Landau, L.D., and Lifshitz, E.M. (1988) 'Hydrodynamics,' Nauka, Moscow, p. 497 (in Russian).

Lipatov, A.S., and Syrovatskii, I.N. (1987) 'Numerical simulation of cometary quasiparallel shocks,' Kosmicheskie Issledovaniya 25, 952-957.

McKenna-Lawlor, S., Kirsch, E., O'Sullivan, D., Thompson, A., and Wenzel, K.P. (1986) 'Energetic ions in the environment of comet Halley,' Nature 321, 347-349.

Mukai, T., Miyake, W., Terasawa, T., Kitayama, M., and Hirao, K. (1986) 'Plasma observations by Suisei of solar wind interaction with comet Halley,' Nature 321, 299-303.

Neugebauer, M., Lazarus, A.J., Altwegg, K., Balsiger, H., Goldstein, B.E., Goldstein, R., Neubauer, F.M., Rosenbauer, H., Schwenn, R., Shelley, E.G., and Ungstrup, E. (1987) 'The pick-up of cometary protons by the solar wind,' Astron. Astrophys. 187, 21.

Neugebauer, M., Lazarus, A.J., Balsiger, H., Fuselier, S.A., Neubauer, F.M., and Rosenbauer, H. (1989a) 'The velocity distributions of cometary protons picked up by the solar wind,' J. Geophys. Res. 94, 5227-5239.

Neugebauer, M., Neubauer, F.M., Balsiger, H., Fuselier, S.A., Goldstein, B.E., Goldstein, R., Mariani, F., Rosenbauer, H., Schwenn, R., and Sehhely, E.G. (1989b) 'The variation of protons, alpha particles and the magnetic field across the bow shock of comet Halley,' J. Geophys. Res., in press. 
Omidi, N., and Winske, D. (1987) 'A kinetic study of solar wind mass loading and cometary bow shocks,' J. Geophys. Res. 92, 1340-1349.

Omidi, N., and Winske, D. (1988) 'Subcritical dispersive shock waves upstream of planetary bow shocks and at comet Giacobini-Zinner,' Geophys. Res. Lett. 15, 1303-1306.

Reme, H., Sauvaud, J.A., d'Uston, C., Cotin, F., Cros, A., Anderson, K.A., Carlson, C.W., Curtis, D.W., Lin, R.P., Mendis, D.A., Korth, A., and Richter, A.K. (1986) 'Comet Halley - solar wind interaction from electron measurements aboard Giotto,' Nature 321, 349-352.

Riedler, W., Schwingenshuh, K., Yeroshenko, Ye.G., Styashkin, V.A., and Russell, C.T. (1986) 'Magnetic field observations in comet Halley's coma,' Nature 321, 288-290.

Sagdeev, R.Z., and Galeev, A.A. (1969) 'Nonlinear Plasma Theory,' Benjamin, New York Amsterdam, p. 54.

Sagdeev, R.Z., and Shafranov, V.D. (1961) 'Instabilities of an anisotropic plasma,' Sov. Phys. JETP 12,130 .

Sagdeev, R.Z., Shapiro, V.D., Shevchenko, V.I., and Szego, K. (1986) 'MHD turbulence in the solar wind-comet interaction region,' Geophys. Res. Lett. 13, 85-88.

Scarf, F.L. (1989) 'Plasma wave observations at comets Giacobini-Zinner and Halley,' in B.T. Tsurutani and H. Oya (eds.), Plasma Waves and Instabilities at Comets and in Magnetospheres, AGU, Washington D.C., pp. 31-40.

Scarf, F.L., Coroniti, F.V., Kennel, C.F., Gurnett, D.A., Ip, W.-H., and Smith, E.J. (1986) 'Plasma wave observations at comet Giacobini-Zinner,' Science 232, 377-381.

Scarf, F.L., Coroniti, F.V., Kennel, C.F., Gurnett, D.A., Ip, W.-H., and Smith, E.J. (1987) 'Observations of cometary plasma-wave phenomena,' Astron. Astrophys. 187, 109-116.

Schmidt, H.U., and Wegmann, R. (1982) 'Plasma flow and magnetic fields in comets,' in L.L. Wilkening (ed.), Comets, University of Arizona Press, Tucson, pp. 538-560.

Skilling, J. (1975) 'Cosmic ray streaming, I. Effect of Alfven waves on particles,' Mon. Not. R. Astron. Soc. 172, 557.

Smith, E.J., Tsurutani, B.T., Slavin, J.A., Jones, D.E., Siscoe, G.L., and Mendis, D.A. (1986) 'International Cometary Explorer encounter with Giacobini-Zinner: Magnetic field observations,' Science 232, 382-385.

Somogyi, A.J., Gringauz, K.I., Szego, K., Szabo, L., Kozma, Gy., Remizov, A.P., Ero, J., Jr., Klimenko, I.N., Szucs, I.T., Verigin, M.I., Windberg, J., Cravens, T.E., Dyachkov, A., Erdos, G., Farago, M., Gombosi, T.I., Kecskemety, K., Keppler, E., Kovacs, T., Konder, A., Logachov, Yu.I., Lobonyai, L., Marsden, R., Redl, R., Richter, A.K., Stolpovskii, V.G., Szabo, J., Szentpetery, I., Szepesvary, A., Tatrallyay, M., Varga, A., Vladimirova, G.A., Wenzel, K.P., and Zaradny, A. (1986) 'First observations of energetic particles near comet Halley,' Nature 321, $285-288$.

Tsurutani, B.T., and Smith, E.J. (1986a) 'Strong hydromagnetic turbulence associated with comet Giacobini-Zinner,' Geophys. Res. Lett. 13, 259-262.

Tsurutani, B.T., and Smith, E.J. (1986b) 'Hydromagnetic waves and instabilities associated with cometary ion pickup: ICE observations,' Geophys. Res. Lett. 13, 263-266.

Tsurutani, B.T., Thorne, R.M., Smith, E.J., Gosling, J.T., and Matsumoto, H. (1987) 'Steepened magnetosonic waves at comet Giaconibi-Zinner,' J. Geophys. Res. 92, 11074-11082.

Vaisberg, O.L., Russell, C.T., Luhman, J.G., and Schwingenshuh, K. (1989) 'Small scale irregularities in comet Halley's plasma mantle: An attempt at self-consistent analysis of plasma and magnetic field data,' Geophys. Res. Lett. 16, 5-8.

Wallis, M.K. (1971) 'Shock-free deceleration of the solar wind?', Nature 233, 23-25.

Wallis, M.K., and Ong, R.S.B. (1975) 'Strongly-cooled ionizing plasma flows with application to Venus,' Planet. Space Sci. 23, 713-721.

Wentzel, D.G. (1974) 'Cosmic ray propagation in the Galaxy: Collective effects,' Ann. Rev. Astron. Astrophys. 12, 71-96. 
Wegmann, R., Schmidt, H.U., Huebner, W.F., and Boice, D.C. (1987) 'Cometary MHD and chemistry,' Astron. Astrophys. 187, 339-350.

Wilken, B., Jockers, K., Studemann, W., Rosenbauer, H., Thomson, M.F., Johnstone, A., Formisano, V., Amata, E., Winningham, J.D., and Borg, H. (1986) 'Energetic cometary water group ions at Halley's bow shock: Observations with the Giotto ion spectrometer IIS,' in Proc. 20th ESLAB Symp. on the Exploration of Halley's Comet, ESA SP-250, pp. 271-276.

Winske, D., and Gary, S.P. (1986) 'Electromagnetic instabilities driven by cool heavy ion beams,' J. Geophys. Res. 91, 6825.

Winske, D., Wu, C.S., Li, Y.Y., Mou, Z.Z., and Guo, S.Y. (1985) 'Coupling of newborn ions to the solar wind by electromagnetic instabilities and their interaction with the bow shock,' J. Geophys. Res. 90, 2713-2726.

Wu, C.S., and Davidson, R.C. (1972) 'Electromagnetic instabilities produced by neutral particle ionization in interplanetary space,' J. Geophys. Res. 77, 5399-5406.

Wu, C.S., and Hartle, R.E. (1974) 'Further remarks on plasma instabilities produced by ions born in the solar wind,' J. Geophys. Res. 79, 283-285. 